\title{
Some geometric aggregation operators based on log-normally distributed random variables
}

\author{
Xin-Fan Wang \\ School of Science, Hunan University of Technology, Zhuzhou 412007, China \\ Jian-Qiang Wang* \\ School of Business, Central South University, Changsha 410083, China \\ Sheng-Yue Deng \\ School of Science, Hunan University of Technology, Zhuzhou 412007, China
}

Received 9 July 2013

Accepted 25 January 2014

\begin{abstract}
The weighted geometric averaging (WGA) operator and the ordered weighted geometric (OWG) operator are two of most basic operators for aggregating information. But these two operators can only be used in situations where the given arguments are exact numerical values. In this paper, we first propose some new geometric aggregation operators, such as the log-normal distribution weighted geometric (LNDWG) operator, log-normal distribution ordered weighted geometric (LNDOWG) operator and log-normal distribution hybrid geometric (LNDHG) operator, which extend the WGA operator and the OWG operator to accommodate the stochastic uncertain environment in which the given arguments are log-normally distributed random variables, and establish various properties of these operators. Then, we apply the LNDWG operator and the LNDHG operator to develop an approach for solving multi-criteria group decision making (MCGDM) problems, in which the criterion values take the form of log-normally distributed random variables and the criterion weight information is known completely. Finally, an example is given to illustrate the feasibility and effectiveness of the developed method.
\end{abstract}

Keywords: Multi-criteria decision making; log-normal distribution; information fusion; LNDWG operator; LNDOWG operator; LNDHG operator

\section{Introduction}

Information aggregation operators play an important role in multi-criteria decision making (MCDM). As we know, the weighted geometric averaging (WGA) operator $^{1}$ and the ordered weighted geometric (OWG) operator $^{2}$ are two of most common operators for aggregating arguments. The WGA operator first weights all the given arguments and then aggregates all these weighted arguments into a collective one. The OWG operator first reorders all the given arguments in descending order and then weights these ordered arguments, and finally aggregates all these ordered weighted arguments into a collective one. But the WGA operator and the OWG operator can only be used in situations where the given arguments are exact numerical values.

\footnotetext{
*Corresponding author. Tel.: +86-731-88830594. E-mail: jqwang@csu.edu.cn (Jian-Qiang Wang).
} 
In the last decades, the WGA operator and the OWG operator have been extended to accommodate the fuzzy and uncertain situations. Xu et al. ${ }^{3-5}$ extended these two operators to accommodate the situations where the input arguments are fuzzy numbers, for example, $\mathrm{Xu}^{3}$ proposed the fuzzy ordered weighted geometric (FOWG) operator, $\mathrm{Xu}$ and $\mathrm{Da}^{4}$ proposed the uncertain ordered weighted geometric (UOWG) operator, Wang and Yang $^{5}$ proposed the trapezoidal fuzzy ordered weighted geometric (TFOWG) operator; Xu et al. ${ }^{6-11}$ extended these two operators to accommodate the situations where the input arguments are intuitionistic fuzzy numbers or extended intuitionistic fuzzy numbers, for instance, $\mathrm{Xu}$ and $\mathrm{Yager}^{6}$ proposed the intuitionistic fuzzy weighted geometric (IFWG) operator, intuitionistic fuzzy ordered weighted geometric (IFOWG) operator and intuitionistic fuzzy hybrid geometric (IFHG) operator, $\mathrm{Wei}^{7}$ proposed the dynamic intuitionistic fuzzy weighted geometric (DIFWG) operator and uncertain dynamic intuitionistic fuzzy weighted geometric (UDIFWG) operator, Tan ${ }^{8}$ proposed the generalized intuitionistic fuzzy ordered geometric averaging (GIFOGA) operator, $\mathrm{Xu}^{9}$ and $\mathrm{Xu}$ and $\mathrm{Chen}^{10}$ developed the interval-valued intuitionistic fuzzy weighted geometric (IIFWG) operator, intervalvalued intuitionistic fuzzy ordered weighted geometric (IIFOWG) operator and interval-valued intuitionistic fuzzy hybrid geometric (IIFHG) operator, Wang ${ }^{11}$ proposed the fuzzy number intuitionistic fuzzy weighted geometric (FIFWG) operator, fuzzy number intuitionistic fuzzy ordered weighted geometric (FIFOWG) operator and fuzzy number intuitionistic fuzzy hybrid geometric (FIFHG) operator; Xu et al. ${ }^{12-15}$ extended these two operators to accommodate the situations where the input arguments are linguistic variables or uncertain linguistic variables, for example, $\mathrm{Xu}^{12}$ proposed the linguistic weighted geometric averaging (LWGA) operator, linguistic ordered weighted geometric averaging (LOWGA) operator and linguistic hybrid geometric averaging (LHGA) operator, $\mathrm{Wei}^{13}$ proposed the extended 2-tuple weighted geometric (ET-WG) operator and extended 2-tuple ordered weighted geometric (ET-OWG) operator, $\mathrm{Xu}^{14}$ proposed the uncertain linguistic weighted geometric mean (ULWGM) operator and uncertain linguistic ordered weighted geometric (ULOWG) operator, Wei ${ }^{15}$ proposed the uncertain linguistic hybrid geometric mean (ULHGM) operator, which is based on the ULWGM operator and the ULOWG operator. In addition, they established various properties of these operators and applied them to solve MCDM problems.

In some MCDM situations, the given arguments take the form of random variables under stochastic uncertain environment ${ }^{16-22}$. However, at present, there is few research related to the aggregation operators for aggregating arguments which are in the form of random variables, except that Wang and Yang ${ }^{23}$ extended the weighted arithmetic averaging (WAA) operator ${ }^{24,25}$ to accommodate the situations where the input arguments are normally distributed random variables, and presented the normal distribution number weighted arithmetic averaging (NDNWAA) operator and the dynamic normal distribution number weighted arithmetic averaging (DNDNWAA) operator.

In addition, in stochastic MCDM problems, normal distribution with bell-shaped curve is usually used to describe the random variation that occurs in the criterion value, and the criterion value is commonly characterized by two parameters: the expected value and the standard deviation $^{16,26,27}$. However, many measurements of criterion values show a more or less skewed distribution. Particularly, skewed distributions are common when expected values are low, variances large, and values cannot be negative. Such skewed distributions often approximately fit the log-normal distribution $^{28,}{ }^{29}$. Moreover, a variable might be distributed as log-normally if it can be thought of as the multiplicative product of a large number of independent random variables each of which is positive. The lognormal distribution is a continuous probability distribution of a random variable whose logarithm is normally distributed ${ }^{28,30}$, and it can model many instances, such as the loss of investment risk, the change in price distribution of a stock, and the failure rates in product tests and so on ${ }^{28,31-33}$. Therefore, in real-life, there are many stochastic MCDM problems in which the criterion values take the form of log-normally distributed random variables. At present, the stochastic MCDM problems, in which the criterion values take the form of normally distributed random variables, have attracted lots of attentions from researchers ${ }^{16-23}$. But regarding to the stochastic MCDM problems, in which the criterion values take the form of log-normally distributed random variables, there is still few related research. It is worthy of pointing out that, in many stochastic MCDM problems with log-normally 
distributed random variables, we need to aggregate the given log-normally distributed random variables into a single one. In such case, information fusion techniques are necessary. In this paper, based on the WGA operator and the OWG operator, we shall develop some new geometric aggregation operators for aggregating arguments which take the form of log-normally distributed random variables, and apply them to solve multi-criteria group decision making (MCGDM) problems in which the criterion values are in the form of log-normally distributed random variables and the criterion weight information is known completely.

In order to do that, this paper is organized as follows. In Section 2, we introduce some basic concepts of lognormal distribution. In Section 3, we extend the WGA operator and the OWG operator to accommodate the situations where the input arguments take the form of log-normally distributed random variables, propose some new geometric aggregation operators, such as the log-normal distribution weighted geometric (LNDWG) operator, log-normal distribution ordered weighted geometric (LNDOWG) operator and log-normal distribution hybrid geometric (LNDHG) operator, and establish various properties of these operators. In Section 4, we apply the LNDWG operator and the LNDHG operator to develop an approach for solving the MCGDM problems, in which the criterion values take the form of log-normally distributed random variables and the criterion weight information is known completely. In Section 5, we provide an illustrative example to demonstrate the feasibility and effectiveness of the developed method. Finally, we conclude the paper in Section 6.

\section{Preliminaries}

The normal distribution is a continuous probability distribution defined by the following probability density function, known as the Gaussian function or informally the bell curve ${ }^{34}$ :

$$
f(x)=\frac{1}{\sqrt{2 \pi} \sigma} e^{-\frac{(x-\mu)^{2}}{2 \sigma^{2}}},-\infty<x<+\infty
$$

where $\mu$ is the expectation, $\sigma>0$ is the standard deviation, and $\sigma^{2}$ is the variance. Generally, we use $X \sim N\left(\mu, \sigma^{2}\right) \quad$ as a mathematical expression meaning that $X$ is distributed normally with the expectation $\mu$ and the variance $\sigma^{2}$.

The log-normal distribution is a probability distribution of a random variable whose logarithm is normally distributed ${ }^{28}$, that is, if $\ln Y \sim N\left(\mu, \sigma^{2}\right)$, then $Y$ has a log-normal distribution. If $Y$ is distributed log-normally with parameters $\mu$ and $\sigma$, then we write $Y \sim \log -N\left(\mu, \sigma^{2}\right)$. For convenience, we call $\beta=\log -N\left(\mu, \sigma^{2}\right)$ a log-normal distribution, and let $\Theta$ be a set of all log-normal distributions.

Definition $1^{28}$ Let $\beta_{1}=\log -N\left(\mu_{1}, \sigma_{1}^{2}\right)$ and $\beta_{2}=\log -N\left(\mu_{2}, \sigma_{2}{ }^{2}\right)$ be two log-normal distributions, then

(1) $\beta_{1} \otimes \beta_{2}=\log -N\left(\mu_{1}+\mu_{2}, \sigma_{1}^{2}+\sigma_{2}^{2}\right)$;

(2) $\beta_{1}^{a}=\log -N\left(a \mu_{1}, a^{2} \sigma_{1}^{2}\right), a \neq 0$.

It's easy to prove that all operational results are still log-normal distributions, and according to these two operational laws, we have the following.

(1) $\beta_{1} \otimes \beta_{2}=\beta_{2} \otimes \beta_{1}$;

(2) $\left(\beta_{1} \otimes \beta_{2}\right) \otimes \beta_{3}=\beta_{1} \otimes\left(\beta_{2} \otimes \beta_{3}\right)$;

(3) $\left(\beta_{1} \otimes \beta_{2}\right)^{a}=\beta_{1}^{a} \otimes \beta_{2}^{a}, a \neq 0$;

(4) $\beta_{1}^{a_{1}} \otimes \beta_{1}^{a_{2}}=\beta_{1}^{a_{1}+a_{2}}, a_{1}, a_{2} \neq 0$.

Furthermore, if $\beta=\log -N\left(\mu, \sigma^{2}\right)$ is a log-normal distribution, then its expected value $\mu_{\log }(\beta)$ and standard deviation $\sigma_{\log }(\beta)$ can be calculated by the following formulas ${ }^{28}$ :

$$
\begin{gathered}
\mu_{\log }(\beta)=e^{\mu+\frac{1}{2} \sigma^{2}} \\
\sigma_{\log }(\beta)=e^{\mu+\frac{1}{2} \sigma^{2}} \sqrt{e^{\sigma^{2}}-1}
\end{gathered}
$$

Therefore, by using the relation between expectation and variance in statistics, in the following, we propose a method for the comparison between two log-normal distributions, which is based on the expected value $\mu_{\log }(\beta)$ and the standard deviation $\sigma_{\log }(\beta)$. 
Definition 2 Let $\beta_{1}=\log -N\left(\mu_{1}, \sigma_{1}^{2}\right)$ and $\beta_{2}=\log -N\left(\mu_{2}, \sigma_{2}^{2}\right) \quad$ be two log-normal distributions, then

(1) If $\mu_{\log }\left(\beta_{1}\right)<\mu_{\log }\left(\beta_{2}\right)$, then $\beta_{1}$ is smaller than $\beta_{2}$, denoted by $\beta_{1}<\beta_{2}$;

(2) If $\mu_{\log }\left(\beta_{1}\right)=\mu_{\log }\left(\beta_{2}\right)$, then

(1) If $\sigma_{\log }\left(\beta_{1}\right)=\sigma_{\log }\left(\beta_{2}\right)$, then $\beta_{1}$ is equal to $\beta_{2}$, denoted by $\beta_{1}=\beta_{2}$;

(2) If $\sigma_{\log }\left(\beta_{1}\right)<\sigma_{\log }\left(\beta_{2}\right)$, then $\beta_{1}$ is bigger than $\beta_{2}$, denoted by $\beta_{1}>\beta_{2}$;

(3) If $\sigma_{\log }\left(\beta_{1}\right)>\sigma_{\log }\left(\beta_{2}\right)$, then $\beta_{1}$ is smaller than $\beta_{2}$, denoted by $\beta_{1}<\beta_{2}$.

\section{Log-normal distribution geometric aggregation operators}

\subsection{The $L N D W G$ and $L N D O W G$ operators}

In what follows, based on Definition 1, we propose some new geometric aggregation operator for aggregating log-normal distribution information, such as the LNDWG operator and the LNDOWG operator.

\section{Definition 3 Let $\quad \beta_{j}=\log -N\left(\mu_{j}, \sigma_{j}{ }^{2}\right)$} $(j=1,2, \cdots, n)$ be a collection of log-normal distributions, and let LNDWG: $\Theta^{n} \rightarrow \Theta$, if

$$
\begin{aligned}
\operatorname{LNDWG}_{w}\left(\beta_{1}, \beta_{2}, \cdots, \beta_{n}\right) & = \\
& \beta_{1}^{w_{1}} \otimes \beta_{2}{ }^{w_{2}} \otimes \cdots \otimes \beta_{n}{ }^{w_{n}}
\end{aligned}
$$

then LNDWG is called the log-normal distribution weighted geometric operator (LNDWG) of dimension $n$, where $\boldsymbol{w}=\left(w_{1}, w_{2}, \cdots, w_{n}\right)^{T}$ is the weight vector of $\beta_{j}(j=1,2, \cdots, n)$, with $w_{j} \geq 0$ and $\sum_{j=1}^{n} w_{j}=1$. Especially, if $\boldsymbol{w}=(1 / n, 1 / n, \cdots, 1 / n)^{T}$, then the LNDWG operator is reduced to the log-normal distribution geometric averaging (LNDGA) operator of dimension $n$, which is defined as:

$$
\operatorname{LNDGA}_{w}\left(\beta_{1}, \beta_{2}, \cdots, \beta_{n}\right)=
$$

$$
\left(\beta_{1} \otimes \beta_{2} \otimes \cdots \otimes \beta_{n}\right)^{\frac{1}{n}}
$$

Theorem 1 Let $\beta_{j}=\log -N\left(\mu_{j}, \sigma_{j}{ }^{2}\right)$ ( $j=1,2, \cdots, n)$ be a collection of log-normal distributions and $\boldsymbol{w}=\left(w_{1}, w_{2}, \cdots, w_{n}\right)^{T}$ be the weight vector of $\beta_{j} \quad(j=1,2, \cdots, n)$, with $w_{j} \geq 0$ and $\sum_{j=1}^{n} w_{j}=1$, then their aggregated result by using the LNDWG operator is also a log-normal distribution, and

$$
\begin{aligned}
& \operatorname{LNDWG}_{w}\left(\beta_{1}, \beta_{2}, \cdots, \beta_{n}\right)= \\
& \quad \log -N\left(\sum_{j=1}^{n} w_{j} \mu_{j}, \sum_{j=1}^{n} w_{j}^{2} \sigma_{j}^{2}\right)
\end{aligned}
$$

Proof: Obviously, the aggregated result by using the LNDWG operator is also a log-normal distribution. In the following, we prove Eq. (5) by using mathematical induction on $n$.

(1) For $n=2$ : since

$$
\begin{aligned}
& \beta_{1}^{w_{1}}=\log -N\left(w_{1} \mu_{1}, w_{1}^{2} \sigma_{1}^{2}\right) \\
& \beta_{2}^{w_{2}}=\log -N\left(w_{2} \mu_{2}, w_{2}^{2} \sigma_{2}^{2}\right)
\end{aligned}
$$

Then

$$
\begin{aligned}
& \operatorname{LNDWG}_{w}\left(\beta_{1}, \beta_{2}\right)=\beta_{1}^{w_{1}} \otimes \beta_{2}^{w_{2}} \\
& =\log -N\left(w_{1} \mu_{1}+w_{2} \mu_{2}, w_{1}^{2} \sigma_{1}^{2}+w_{2}^{2} \sigma_{2}^{2}\right) \\
& =\log -N\left(\sum_{j=1}^{2} w_{j} \mu_{j}, \sum_{j=1}^{2} w_{j}^{2} \sigma_{j}^{2}\right) .
\end{aligned}
$$

(2) If Eq. (5) holds for $n=k$, that is $\operatorname{LNDWG}_{w}\left(\beta_{1}, \beta_{2}, \cdots, \beta_{k}\right)=$

$$
\log -N\left(\sum_{j=1}^{k} w_{j} \mu_{j}, \sum_{j=1}^{k} w_{j}^{2} \sigma_{j}^{2}\right)
$$

Then, when $n=k+1$, by Definition 1, we have

$$
\begin{aligned}
& \operatorname{LNDWG}_{w}\left(\beta_{1}, \beta_{2}, \cdots, \beta_{k}, \beta_{k+1}\right) \\
&=\log -N\left(\sum_{j=1}^{k} w_{j} \mu_{j}, \sum_{j=1}^{k} w_{j}^{2} \sigma_{j}^{2}\right) \otimes \\
&\left(\log -N\left(\mu_{k+1}, \sigma_{k+1}^{2}\right)\right)^{w_{k+1}} \\
&= \log -N\left(\sum_{j=1}^{k} w_{j} \mu_{j}, \sum_{j=1}^{k} w_{j}^{2} \sigma_{j}^{2}\right) \otimes
\end{aligned}
$$




$$
\begin{aligned}
\log -N\left(w_{k+1} \mu_{k+1}, w_{k+1}^{2} \sigma_{k+1}^{2}\right) \\
=\log -N\left(\sum_{j=1}^{k} w_{j} \mu_{j}+w_{k+1} \mu_{k+1},\right. \\
\left.\sum_{j=1}^{k} w_{j}^{2} \sigma_{j}^{2}+w_{k+1}^{2} \sigma_{k+1}^{2}\right) \\
=\log -N\left(\sum_{j=1}^{k+1} w_{j} \mu_{j}, \sum_{j=1}^{k+1} w_{j}^{2} \sigma_{j}^{2}\right) .
\end{aligned}
$$

i.e. Eq. (5) holds for $n=k+1$.

Thus, based on (1) and (2), Eq. (5) holds for all $n \in N$, which completes the proof of Theorem 1 .

Example 1. Let $\beta_{1}=\log -N\left(2.06,0.20^{2}\right)$, $\beta_{2}=\log -N\left(2.21,0.18^{2}\right) \quad, \quad \beta_{3}=\log -N(1.39$, $\left.0.12^{2}\right)$ and $\beta_{4}=\log -N\left(1.93,0.21^{2}\right)$ be four lognormal distributions, and $\boldsymbol{w}=(0.2,0.3,0.1,0.4)^{T}$ be the weight vector of $\beta_{j}(j=1,2,3,4)$, then

$$
\begin{aligned}
& \mathrm{LNDWG}_{w}\left(\beta_{1}, \beta_{2}, \beta_{3}, \beta_{4}\right) \\
&=\log -N(0.2 \cdot 2.06+0.3 \cdot 2.21+ \\
& 0.1 \cdot 1.39+0.4 \cdot 1.93,0.2^{2} \cdot 0.20^{2}+ \\
&\left.0.3^{2} \cdot 0.18^{2}+0.1^{2} \cdot 0.12^{2}+0.4^{2} \cdot 0.21^{2}\right) \\
&= \log -N\left(1.986,0.108^{2}\right) .
\end{aligned}
$$

Theorem 2 (Properties of LNDWG) Let $\beta_{j}=\log -N\left(\mu_{j}, \sigma_{j}{ }^{2}\right) \quad(j=1,2, \cdots, n) \quad$ be $\quad$ a collection of log-normal distributions, and $\boldsymbol{w}=\left(w_{1}, w_{2}, \cdots, w_{n}\right)^{T}$ be the weight vector of $\beta_{j}$ $(j=1,2, \cdots, n)$, with $w_{j} \in[0,1]$ and $\sum_{j=1}^{n} w_{j}=1$, then we have the following.

(1) (Idempotency): If all $\beta_{j}(j=1,2, \cdots, n)$ are equal, i.e. $\beta_{j}=\beta$ for all $j$, then

$$
\operatorname{LNDWG}_{w}\left(\beta_{1}, \beta_{2}, \cdots, \beta_{n}\right)=\beta
$$

(2) (Boundary) :

$$
\beta^{-} \leq \mathrm{LNDWG}_{w}\left(\beta_{1}, \beta_{2}, \cdots, \beta_{n}\right) \leq \beta^{+}
$$

where $\beta^{-}=\min _{j}\left\{\beta_{j}\right\}, \beta^{+}=\max _{j}\left\{\beta_{j}\right\}$.
(3) (Monotonicity): Let $\beta_{j}^{*}(j=1,2, \cdots, n)$ be a collection of log-normal distributions. If $\beta_{j} \leq \beta_{j}^{*}$ for all $j$, then

$$
\begin{aligned}
& \operatorname{LNDWG}_{w}\left(\beta_{1}, \beta_{2}, \cdots, \beta_{n}\right) \leq \\
& \operatorname{LNDWG}_{w}\left(\beta_{1}^{*}, \beta_{2}^{*}, \cdots, \beta_{n}^{*}\right)
\end{aligned}
$$

Definition 4. Let $\beta_{j}=\log -N\left(\mu_{j}, \sigma_{j}{ }^{2}\right)$ ( $j=1,2, \cdots, n)$ be a collection of log-normal distributions. A log-normal distribution ordered weighted geometric (LNDOWG) operator of dimension $n$ is a mapping LNDOWG: $\Theta^{n} \rightarrow \Theta$, that has an associated weight vector $\boldsymbol{\omega}=\left(\omega_{1}, \omega_{2}, \cdots, \omega_{n}\right)^{T}$ such that $\omega_{j} \geq 0$ and $\sum_{j=1}^{n} \omega_{j}=1$. Furthermore,

$$
\begin{aligned}
\text { LNDOWG }_{\omega}( & \left.\beta_{1}, \beta_{2}, \cdots, \beta_{n}\right)= \\
& \beta_{\tau(1)}^{\omega_{1}} \otimes \beta_{\tau(2)}^{\omega_{2}} \otimes \cdots \otimes \beta_{\tau(n)}^{\omega_{n}}
\end{aligned}
$$

where $(\tau(1), \tau(2), \cdots, \tau(n))$ is a permutation of $(1,2, \cdots, n)$ such that $\beta_{\tau(j-1)} \geq \beta_{\tau(j)}$ for all $j$. Especially, if $\omega=(1 / n, 1 / n, \cdots, 1 / n)^{T}$, then the LNDOWG operator is reduced to the LNDGA operator.

Similar to Theorem 1, we have the following.

Theorem 3. Let $\beta_{j}=\log -N\left(\mu_{j}, \sigma_{j}{ }^{2}\right)$ ( $j=1,2, \cdots, n)$ be a collection of log-normal distributions, then their aggregated result using the LNDOWG operator is also a log-normal distribution, and

$$
\begin{aligned}
& \operatorname{LNDOWG}_{\omega}\left(\beta_{1}, \beta_{2}, \cdots, \beta_{n}\right)= \\
& \log -N\left(\sum_{j=1}^{n} \omega_{j} \mu_{\tau(j)}, \sum_{j=1}^{n} \omega_{j}^{2} \sigma_{\tau(j)}^{2}\right)
\end{aligned}
$$

where $\boldsymbol{\omega}=\left(\omega_{1}, \omega_{2}, \cdots, \omega_{n}\right)^{T}$ is the weight vector related to the LNDOWG operator, with $\omega_{j} \geq 0$ and $\sum_{j=1}^{n} \omega_{j}=1$, which can be determined similar to the OWA weights ${ }^{35}$. 
Example 2. Let $\beta_{1}=\log -N\left(2.06,0.20^{2}\right)$, $\beta_{2}=\log -N\left(2.21,0.18^{2}\right) \quad, \quad \beta_{3}=\log -N(1.39$, $\left.0.12^{2}\right)$ and $\beta_{4}=\log -N\left(1.93,0.21^{2}\right)$ be four lognormal distributions. Then by Eq. (1), we can calculate the expected values of $\beta_{i}(j=1,2,3,4)$ :

$$
\begin{aligned}
& \mu_{\log }\left(\beta_{1}\right)=8.0045, \mu_{\log }\left(\beta_{2}\right)=9.2646, \\
& \mu_{\log }\left(\beta_{3}\right)=4.0439, \mu_{\log }\left(\beta_{4}\right)=7.0431 .
\end{aligned}
$$

Since

$$
\mu_{\log }\left(\beta_{2}\right)>\mu_{\log }\left(\beta_{1}\right)>\mu_{\log }\left(\beta_{4}\right)>\mu_{\log }\left(\beta_{3}\right)
$$

Thus

$$
\begin{aligned}
& \beta_{\tau(1)}=\beta_{2}=N\left(2.21,0.18^{2}\right), \\
& \beta_{\tau(2)}=\beta_{1}=N\left(2.06,0.20^{2}\right), \\
& \beta_{\tau(3)}=\beta_{4}=N\left(1.93,0.21^{2}\right), \\
& \beta_{\tau(4)}=\beta_{3}=N\left(1.39,0.12^{2}\right) .
\end{aligned}
$$

Suppose that $\boldsymbol{\omega}=(0.155,0.345,0.345$

$0.155)^{T}$ (derived by the normal distribution based method ${ }^{[35]}$ ) is the weight vector of the LNDOWG operator, then by Eq. (10), we get

$$
\begin{aligned}
& \mathrm{LNDOWG}_{w}\left(\beta_{1}, \beta_{2}, \beta_{3}, \beta_{4}\right) \\
&=\log -N(0.155 \cdot 2.21+0.345 \cdot 2.06+ \\
& 0.345 \cdot 1.93+0.155 \cdot 1.39, \\
& 0.155^{2} \cdot 0.18^{2}+0.345^{2} \cdot 0.20^{2}+ \\
&\left.0.345^{2} \cdot 0.21^{2}+0.155^{2} \cdot 0.12^{2}\right) \\
&= \log -N\left(1.9346,0.1055^{2}\right) .
\end{aligned}
$$

The LNDOWG operator has the following properties similar to those of the LNDWG operator.

Theorem 4 (Properties of LNDOWG) Let $\beta_{j}=\log -N\left(\mu_{j}, \sigma_{j}{ }^{2}\right) \quad(j=1,2, \cdots, n) \quad$ be $\quad$ a collection of log-normal distributions, and $\boldsymbol{\omega}=\left(\omega_{1}, \omega_{2}, \cdots, \omega_{n}\right)^{T}$ be the weight vector related to the LNDOWG operator, with $\omega_{j} \in[0,1]$ and $\sum_{j=1}^{n} \omega_{j}=1$, then we have the following.

(1) (Idempotency): If all $\beta_{j}(j=1,2, \cdots, n)$ are equal, i.e. $\beta_{j}=\beta$ for all $j$, then

$$
\text { LNDOWG }_{\omega}\left(\beta_{1}, \beta_{2}, \cdots, \beta_{n}\right)=\beta
$$

(2) (Boundary) :

$$
\beta^{-} \leq \operatorname{LNDOWG}_{\omega}\left(\beta_{1}, \beta_{2}, \cdots, \beta_{n}\right) \leq \beta^{+}
$$

where $\beta^{-}=\min _{j}\left\{\beta_{j}\right\}, \beta^{+}=\max _{j}\left\{\beta_{j}\right\}$.

(3) (Monotonicity): Let $\beta_{j}^{*}(j=1,2, \cdots, n)$ be a collection of log-normal distributions. If $\beta_{j} \leq \beta_{j}^{*}$ for all $j$, then

$$
\begin{aligned}
& \operatorname{LNDOWG}_{\omega}(\left.\beta_{1}, \beta_{2}, \cdots, \beta_{n}\right) \leq \\
& \operatorname{LNDOWG}_{\omega}\left(\beta_{1}^{*}, \beta_{2}^{*}, \cdots, \beta_{n}^{*}\right)
\end{aligned}
$$

(4) (Commutativity): Let $\tilde{\beta}_{j}(j=1,2, \cdots, n)$ be a collection of log-normal distributions, then

$$
\begin{aligned}
& \operatorname{LNDOWG}_{\omega}\left(\beta_{1}, \beta_{2}, \cdots, \beta_{n}\right)= \\
& \operatorname{LNDOWG}_{\omega}\left(\tilde{\beta}_{1}, \tilde{\beta}_{2}, \cdots, \tilde{\beta}_{n}\right)
\end{aligned}
$$

where $\left(\tilde{\beta}_{1}, \tilde{\beta}_{2}, \cdots, \tilde{\beta}_{n}\right)$ is any permutation of $\left(\beta_{1}, \beta_{2}, \cdots, \beta_{n}\right)$.

From Eq. (14), it can be known that the LNDOWG operator has commutativity property that we desire. It is worth noting that the LNDWG operator does not have this property.

Besides the above properties, the LNDOWG operator has the following desirable results.

Theorem 5 Let $\quad \beta_{j}=\log -N\left(\mu_{j}, \sigma_{j}^{2}\right)$ $(j=1,2, \cdots, n)$ be a collection of log-normal distributions, and $\boldsymbol{\omega}=\left(\omega_{1}, \omega_{2}, \cdots, \omega_{n}\right)^{T}$ be the weight vector related to the LNDOWG operator, with $\omega_{j} \in[0,1]$ and $\sum_{j=1}^{n} \omega_{j}=1$, then

(1) If $\boldsymbol{\omega}=(1,0, \cdots, 0)^{T}$, then

$$
\text { LNDOWG }_{\omega}\left(\beta_{1}, \beta_{2}, \cdots, \beta_{n}\right)=\max _{j}\left\{\beta_{j}\right\} \text {. }
$$

(2) If $\boldsymbol{\omega}=(0,0, \cdots, 1)^{T}$, then

$$
\text { LNDOWG }_{\omega}\left(\beta_{1}, \beta_{2}, \cdots, \beta_{n}\right)=\min _{j}\left\{\beta_{j}\right\} \text {. }
$$

(3) If $\omega_{j}=1, \omega_{r}=0$, and $r \neq j$, then

$$
\text { LNDOWG }_{\omega}\left(\beta_{1}, \beta_{2}, \cdots, \beta_{n}\right)=\beta_{\tau(j)}
$$

where $\beta_{\tau(j)}$ is the $j$ th largest of $\beta_{r}(r=1,2, \cdots, n)$. 


\subsection{The LNDHG operator}

From Definition 3 and Definition 4, it can be known that the LNDWG operator weights only the log-normal distributions, whereas the LNDOWG operator weights only the ordered positions of the log-normal distributions instead of weighting the arguments themselves. To overcome this limitation, in what follows, we propose a LNDHG operator, which weights both the given log-normal distributions and their ordered positions.

Definition 5. Let $\beta_{j}=\log -N\left(\mu_{j}, \sigma_{j}^{2}\right)$ $(j=1,2, \cdots, n)$ be a collection of log-normal distributions. A log-normal distribution hybrid geometric (LNDHG) operator of dimension $n$ is a mapping LNDHG: $\Theta^{n} \rightarrow \Theta$, which has an associated vector $\boldsymbol{\omega}=\left(\omega_{1}, \omega_{2}, \cdots, \omega_{n}\right)^{T}$ with $\omega_{j} \geq 0$ and $\sum_{j=1}^{n} \omega_{j}=1$, such that

$$
\begin{aligned}
& \operatorname{LNDHG}_{w, \omega}\left(\beta_{1}, \beta_{2}, \cdots, \beta_{n}\right)= \\
& \quad\left(\beta_{\tau(1)}^{\prime}\right)^{\omega_{1}} \otimes\left(\beta_{\tau(2)}^{\prime}\right)^{\omega_{2}} \otimes \cdots \otimes\left(\beta_{\tau(n)}^{\prime}\right)^{\omega_{n}}
\end{aligned}
$$

where $\beta_{\tau(j)}^{\prime}$ is the $j$ th largest of weighted log-normal distributions $\quad\left(\beta_{1}^{n w_{1}}, \beta_{2}^{n w_{2}}, \cdots, \beta_{n}^{n w_{n}} \quad\right)$, $\boldsymbol{w}=\left(w_{1}, w_{2}, \cdots, w_{n}\right)^{T}$ is the weight vector of $\beta_{j}$ $(j=1,2, \cdots, n)$, with $w_{j} \geq 0$ and $\sum_{j=1}^{n} w_{j}=1$, and $n$ is the balancing coefficient.

Theorem 6. Let $\beta_{j}=\log -N\left(\mu_{j}, \sigma_{j}{ }^{2}\right)$ ( $j=1,2, \cdots, n)$ be a collection of log-normal distributions and $\quad \beta_{\tau(j)}^{\prime}=\log -N\left(\mu_{\tau(j)}^{\prime}, \sigma_{\tau(j)}^{\prime 2}\right)$ $(j=1,2, \cdots, n)$, then

$$
\begin{aligned}
& \mathrm{LNDHG}_{\boldsymbol{w}, \boldsymbol{\omega}}\left(\beta_{1}, \beta_{2}, \cdots, \beta_{n}\right)= \\
& \log -N\left(\sum_{j=1}^{n} \omega_{j} \mu_{\tau(j)}^{\prime}, \sum_{j=1}^{n} \omega_{j}^{2} \sigma_{\tau(j)}^{\prime 2}\right)
\end{aligned}
$$

and the aggregated result derived by using the LNDHG operator is also a log-normal distribution.
Example 3. Let $\beta_{1}=\log -N\left(2.16,0.17^{2}\right)$, $\beta_{2}=\log -N\left(2.21,0.18^{2}\right) \quad, \quad \beta_{3}=\log -N(2.19$, $\left.0.12^{2}\right)$ and $\beta_{4}=\log -N\left(2.03,0.11^{2}\right)$ be four lognormal distributions, and let $\boldsymbol{w}=(0.2,0.3$, $0.3,0.2)^{T}$ be the weight vector of $\beta_{j}$ $(j=1,2,3,4)$. Then by Definition 1 , we can get the weighted log-normal distributions:

$$
\begin{aligned}
& \beta_{1}^{\prime}=\log -N\left(1.728,0.136^{2}\right), \\
& \beta_{2}^{\prime}=\log -N\left(2.652,0.216^{2}\right), \\
& \beta_{3}^{\prime}=\log -N\left(2.628,0.144^{2}\right), \\
& \beta_{4}^{\prime}=\log -N\left(1.624,0.088^{2}\right) .
\end{aligned}
$$

By Eq. (1), we can calculate the expected values of $\beta_{j}^{\prime}(j=1,2,3,4)$ :

$$
\begin{aligned}
& \mu_{\log }\left(\beta_{1}^{\prime}\right)=5.6817, \mu_{\log }\left(\beta_{2}^{\prime}\right)=14.5171, \\
& \mu_{\log }\left(\beta_{3}^{\prime}\right)=13.9904, \mu_{\log }\left(\beta_{4}^{\prime}\right)=5.0930 .
\end{aligned}
$$

Since

$$
\mu_{\log }\left(\beta_{2}^{\prime}\right)>\mu_{\log }\left(\beta_{3}^{\prime}\right)>\mu_{\log }\left(\beta_{1}^{\prime}\right)>\mu_{\log }\left(\beta_{4}^{\prime}\right)
$$

Thus

$$
\begin{aligned}
& \beta_{\tau(1)}^{\prime}=\beta_{2}^{\prime}=N\left(2.652,0.216^{2}\right), \\
& \beta_{\tau(2)}^{\prime}=\beta_{3}^{\prime}=N\left(2.628,0.144^{2}\right), \\
& \beta_{\tau(3)}^{\prime}=\beta_{1}^{\prime}=N\left(1.728,0.136^{2}\right), \\
& \beta_{\tau(4)}^{\prime}=\beta_{4}^{\prime}=N\left(1.624,0.088^{2}\right) .
\end{aligned}
$$

Suppose that $\boldsymbol{\omega}=(0.155,0.345,0.345,0.155)^{T}$ (determined by the normal distribution based method ${ }^{35}$ ) is the weight vector of the LNDHG operator, then by Eq. (16), it follows that

$$
\begin{aligned}
& \mathrm{LNDHG}_{w, \omega}\left(\beta_{1}, \beta_{2}, \beta_{3}, \beta_{4}\right) \\
&=\log -N(0.155 \cdot 2.652+0.345 \cdot 2.628+ \\
& 0.345 \cdot 1.728+0.155 \cdot 1.624, \\
& 0.155^{2} \cdot 0.216^{2}+0.345^{2} \cdot 0.144^{2}+ \\
&\left.0.345^{2} \cdot 0.136^{2}+0.155^{2} \cdot 0.088^{2}\right) \\
&= \log -N\left(2.1656,0.0773^{2}\right) .
\end{aligned}
$$

Theorem 7. The LNDWG operator is a special case of the LNDHG operator.

Proof. Let $\boldsymbol{\omega}=(1 / n, 1 / n, \cdots, 1 / n)^{T}$, then 


$$
\begin{aligned}
& \mathrm{LNDHG}_{w, \omega}\left(\beta_{1}, \beta_{2}, \cdots, \beta_{n}\right) \\
& =\left(\beta_{\tau(1)}^{\prime}\right)^{\omega_{1}} \otimes\left(\beta_{\tau(2)}^{\prime}\right)^{\omega_{2}} \otimes \cdots \otimes\left(\beta_{\tau(n)}^{\prime}\right)^{\omega_{n}} \\
& =\left(\beta_{1}^{\prime} \otimes \beta_{2}^{\prime} \otimes \cdots \otimes \beta_{n}^{\prime}\right)^{\frac{1}{n}} \\
& =\beta_{1}^{w_{1}} \otimes \beta_{2}^{w_{2}} \otimes \cdots \otimes \beta_{n}^{w_{n}} \\
& =\mathrm{LNDWG}_{w}\left(\beta_{1}, \beta_{2}, \cdots, \beta_{n}\right)
\end{aligned}
$$

which completes the proof of Theorem 7 .

Theorem 8. The LNDOWG operator is a special case of the LNDHG operator.

Proof. Let $\boldsymbol{w}=(1 / n, 1 / n, \cdots, 1 / n)^{T}$, then $\beta_{j}^{\prime}=\beta_{j}$, $j=1,2, \cdots, n$, then

$$
\begin{aligned}
& \mathrm{LNDHG}_{w, \omega}\left(\beta_{1}, \beta_{2}, \cdots, \beta_{n}\right) \\
& =\left(\beta_{\tau(1)}^{\prime}\right)^{\omega_{1}} \otimes\left(\beta_{\tau(2)}^{\prime}\right)^{\omega_{2}} \otimes \cdots \otimes\left(\beta_{\tau(n)}^{\prime}\right)^{\omega_{n}} \\
& =\beta_{\tau(1)}^{\omega_{1}} \otimes \beta_{\tau(2)}^{\omega_{2}} \otimes \cdots \otimes \beta_{\tau(n)}^{\omega_{n}} \\
& =\mathrm{LNDOWG}_{\omega}\left(\beta_{1}, \beta_{2}, \cdots, \beta_{n}\right)
\end{aligned}
$$

which completes the proof of Theorem 8 .

Obviously, from Theorem 7 and Theorem 8, we can see that the LNDHG operator weights both the given log-normal distributions and the ordered positions of these arguments, reflects the importance degrees of both the given log-normal distributions and their ordered positions, and generalizes both the LNDWG operator and the LNDOWG operator at the same time.

\section{An application of the LNDWG and LNDHG operators to MCGDM}

In this section, we apply the LNDWG and LNDHG operators to MCGDM based on log-normally distributed random variables.

Let $X=\left\{X_{1}, X_{2}, \cdots, X_{m}\right\}$ be a discrete set of feasible alternatives, $C=\left\{C_{1}, C_{2}, \cdots, C_{n}\right\}$ be the finite set of criteria, and $\boldsymbol{w}=\left(w_{1}, w_{2}, \cdots, w_{n}\right)^{T}$ be the weight vector of $C_{j}(j=1,2, \cdots, n)$ with $w_{j} \geq 0$ and $\sum_{j=1}^{n} w_{j}=1$. Let $D=\left\{d_{1}, d_{2}, \cdots, d_{t}\right\}$ be the set of decision makers, and $\boldsymbol{e}=\left(e_{1}, e_{2}, \cdots, e_{t}\right)^{T}$ be the weight vector of $d_{k}(k=1,2, \cdots, t)$ with $e_{k} \geq 0$ and $\sum_{k=1}^{t} e_{k}=1$. Assume that the decision makers $d_{k}$ ( $k=1,2, \cdots, t)$ represent, respectively, the characteristics of the alternatives $X_{i}(i=1,2, \cdots, m)$ with respect to the criteria $C_{j}(j=1,2, \cdots, n)$ by lognormally distributed random variables $\beta_{i j}^{(k)}=\log -N\left(\mu_{i j}^{(k)},\left(\sigma_{i j}^{(k)}\right)^{2} \quad(\quad i=1,2, \cdots, m \quad ;\right.$ $j=1,2, \cdots, n ; k=1,2, \cdots, t)$, and construct decision matrices $\boldsymbol{R}^{(k)}=\left(\beta_{i j}^{(k)}\right)_{m \times n}(k=1,2, \cdots, t)$.

Based on the above decision information, in the following, we propose a practical procedure to select the most desirable alternative(s).

Step 1 Normalize the decision matrices $\boldsymbol{R}^{(k)}=\left(\beta_{i j}^{(k)}\right)_{m \times n}(k=1,2, \cdots, t)$. Let $C^{b}$ be the set of all benefit criteria and $C^{c}$ be the set of all cost criteria, then we can use the following formulas to transform the decision matrices $\boldsymbol{R}^{(k)}=\left(\beta_{i j}^{(k)}\right)_{m \times n}$ into the corresponding normalized decision matrices $\tilde{\boldsymbol{R}}^{(k)}=\left(\tilde{\beta}_{i j}^{(k)}\right)_{m \times n}=\left(\log -N\left(\tilde{\mu}_{i j}^{(k)},\left(\tilde{\sigma}_{i j}^{(k)}\right)^{2}\right)\right)_{m \times n}$ $(k=1,2, \cdots, t)$ :

$$
\begin{aligned}
& \tilde{\mu}_{i j}^{(k)}=\frac{\mu_{i j}^{(k)}}{\max _{k} \max _{i}\left\{\mu_{i j}^{(k)}\right\}}, C_{j} \in C^{b} \\
& \tilde{\mu}_{i j}^{(k)}=\frac{\min _{k} \min _{i}\left\{\mu_{i j}^{(k)}\right\}}{\mu_{i j}^{(k)}}, C_{j} \in C^{c} \\
& \tilde{\sigma}_{i j}^{(k)}=\frac{\sigma_{i j}^{(k)}}{\max _{k} \max _{i}\left\{\mu_{i j}^{(k)}\right\}}, C_{j} \in C
\end{aligned}
$$

Note that standard deviation is relative to expectation, so the Eq. (19) is suitable for all $C_{j} \in C$.

Step 2 Utilize the LNDWG operator

$$
\tilde{\beta}_{i}^{(k)}=\operatorname{LNDWG}_{w}\left(\tilde{\beta}_{i 1}^{(k)}, \tilde{\beta}_{i 2}^{(k)}, \cdots, \tilde{\beta}_{i n}^{(k)}\right)
$$

to aggregate the criterion values of the $i$ th column of the normalized decision matrices $\tilde{\boldsymbol{R}}^{(k)}=\left(\tilde{\beta}_{i j}^{(k)}\right)_{m \times n}$ and derive the individual overall values $\tilde{\beta}_{i}^{(k)}=\log -N\left(\tilde{\mu}_{i}^{(k)},\left(\tilde{\sigma}_{i}^{(k)}\right)^{2}\right)$ of the alternatives $X_{i}$ 
( $i=1,2, \cdots, m$ ) given by the decision makers $d_{k}$ $(k=1,2, \cdots, t)$, where

$$
\tilde{\mu}_{i}^{(k)}=\sum_{j=1}^{n} w_{j} \tilde{\mu}_{i j}^{(k)},\left(\tilde{\sigma}_{i}^{(k)}\right)^{2}=\sum_{j=1}^{n} w_{j}^{2}\left(\tilde{\sigma}_{i j}^{(k)}\right)^{2}
$$

Step 3 Utilize the LNDHG operator

$$
\begin{aligned}
\tilde{\beta}_{i} & =\operatorname{LNDHG}_{\boldsymbol{e}, \boldsymbol{v}}\left(\tilde{\beta}_{i}^{(1)}, \tilde{\beta}_{i}^{(2)}, \cdots, \tilde{\beta}_{i}^{(t)}\right) \\
& =\left(\tilde{\beta}_{i}^{\prime(\tau(1))}\right)^{v_{1}} \otimes\left(\tilde{\beta}_{i}^{\prime(\tau(2))}\right)^{v_{2}} \otimes \cdots \otimes\left(\tilde{\beta}_{i}^{\prime(\tau(t))}\right)^{v_{t}}
\end{aligned}
$$

to derive the collective overall values $\tilde{\beta}_{i}=\log -N\left(\tilde{\mu}_{i}, \tilde{\sigma}_{i}^{2}\right) \quad$ of the alternatives $X_{i}$ $(i=1,2, \cdots, m)$, where

$$
\tilde{\mu}_{i}=\sum_{k=1}^{t} v_{k} \tilde{\mu}_{i}^{\prime \tau(k)}, \tilde{\sigma}_{i}^{2}=\sum_{k=1}^{t} v_{k}^{2}\left(\tilde{\sigma}_{i}^{\prime \tau(k)}\right)^{2}
$$

$\boldsymbol{v}=\left(v_{1}, v_{2}, \cdots, v_{t}\right)^{T}$ is the weighting vector of the LNDHG operator, with $v_{k} \geq 0$ and $\sum_{k=1}^{t} v_{k}=1$, $\tilde{\beta}_{i}^{\prime(\tau(k))}=\log -N\left(\tilde{\mu}_{i}^{\prime \tau(k)},\left(\tilde{\sigma}_{i}^{\prime \tau(k)}\right)^{2}\right) \quad$ is the $k$ th largest of the weighted log-normal distributions $\left.\left(\tilde{\beta}_{i}^{(1)}\right)^{t t_{1}},\left(\tilde{\beta}_{i}^{(2)}\right)^{t e_{2}}, \cdots,\left(\tilde{\beta}_{i}^{(t)}\right)^{t e_{t}} \quad\right)$,

Table 1. Decision matrix $\boldsymbol{R}^{(1)}$ (unit: ten thousands RMB)

\begin{tabular}{cccc}
\hline & $C_{1}$ & $C_{2}$ & $C_{3}$ \\
\hline$X_{1}$ & $\log -N\left(399,9.2^{2}\right)$ & $\log -N\left(279,7.9^{2}\right)$ & $\log -N\left(139,6.6^{2}\right)$ \\
$X_{2}$ & $\log -N\left(393,10.1^{2}\right)$ & $\log -N\left(269,8.5^{2}\right)$ & $\log -N\left(136,6.1^{2}\right)$ \\
$X_{3}$ & $\log -N\left(368,8.9^{2}\right)$ & $\log -N\left(273,6.8^{2}\right)$ & $\log -N\left(130,6.8^{2}\right)$ \\
$X_{4}$ & $\log -N\left(428,10.9^{2}\right)$ & $\log -N\left(307,7.5^{2}\right)$ & $\log -N\left(166,7.2^{2}\right)$ \\
$X_{5}$ & $\log -N\left(463,9.6^{2}\right)$ & $\log -N\left(313,6.9^{2}\right)$ & $\log -N\left(159,7.5^{2}\right)$ \\
\hline
\end{tabular}

Table 2. Decision matrix $\boldsymbol{R}^{(2)}$ (unit: ten thousands RMB)

\begin{tabular}{cccc}
\hline & $C_{1}$ & $C_{2}$ & $C_{3}$ \\
\hline$X_{1}$ & $\log -N\left(381,9.6^{2}\right)$ & $\log -N\left(251,7.6^{2}\right)$ & $\log -N\left(134,5.5^{2}\right)$ \\
$X_{2}$ & $\log -N\left(385,10.2^{2}\right)$ & $\log -N\left(269,9.3^{2}\right)$ & $\log -N\left(138,6.1^{2}\right)$ \\
$X_{3}$ & $\log -N\left(359,9.3^{2}\right)$ & $\log -N\left(253,8.6^{2}\right)$ & $\log -N\left(135,6.5^{2}\right)$ \\
$X_{4}$ & $\log -N\left(463,10.9^{2}\right)$ & $\log -N\left(309,9.1^{2}\right)$ & $\log -N\left(169,7.5^{2}\right)$ \\
$X_{5}$ & $\log -N\left(455,9.7^{2}\right)$ & $\log -N\left(319,8.9^{2}\right)$ & $\log -N\left(157,8.6^{2}\right)$ \\
\hline
\end{tabular}

Table 3. Decision matrix $\boldsymbol{R}^{(3)}$ (unit: ten thousands RMB)

\begin{tabular}{cccc}
\hline & $C_{1}$ & $C_{2}$ & $C_{3}$ \\
\hline$X_{1}$ & $\log -N\left(379,9.2^{2}\right)$ & $\log -N\left(255,7.9^{2}\right)$ & $\log -N\left(131,5.7^{2}\right)$ \\
$X_{2}$ & $\log -N\left(391,9.8^{2}\right)$ & $\log -N\left(269,9.2^{2}\right)$ & $\log -N\left(136,6.1^{2}\right)$ \\
$X_{3}$ & $\log -N\left(351,10.6^{2}\right)$ & $\log -N\left(257,8.6^{2}\right)$ & $\log -N\left(133,6.7^{2}\right)$ \\
$X_{4}$ & $\log -N\left(362,10.6^{2}\right)$ & $\log -N\left(326,9.3^{2}\right)$ & $\log -N\left(168,7.1^{2}\right)$ \\
$X_{5}$ & $\log -N\left(469,11.7^{2}\right)$ & $\log -N\left(309,8.8^{2}\right)$ & $\log -N\left(158,7.6^{2}\right)$ \\
\hline
\end{tabular}


$(\tau(1), \tau(2), \cdots, \tau(t))$ is a permutation of $(1,2, \cdots, t)$, and $t$ is the balancing coefficient.

Step 4 Utilize Eq. (1) and Eq. (2) to calculate the expected values $\mu_{\log }\left(\tilde{\beta}_{i}\right)$ and the standard deviations $\sigma_{\log }\left(\tilde{\beta}_{i}\right)$ of the collective overall values $\tilde{\beta}_{i}$ $(i=1,2, \cdots, m)$.

Step 5 Use Definition 2 to rank all the alternatives $X_{i}(i=1,2, \cdots, m)$, and then select the best one according to the values $\mu_{\log }\left(\tilde{\beta}_{i}\right)$ and $\sigma_{\log }\left(\tilde{\beta}_{i}\right)$ $(i=1,2, \cdots, m)$.

\section{Illustrative example}

Let us suppose that there is an investment company, which wants to invest a total amount of money in the best option (adapted from Ref. 17). There is a panel with five possible companies in which to invest the money: (1) $X_{1}$ is an arms company; (2) $X_{2}$ is a computer company; (3) $X_{3}$ is a food company; (4) $X_{4}$ is an auto company; and (5) $X_{5}$ is a TV company. The criteria to be considered in the selection process are the following: (1) $C_{1}$ : cost; (2) $C_{2}$ : net present value; and (3) $C_{3}:$ loss, whose weighting vector is $\boldsymbol{w}=(0.35,0.37,0.28)^{T}$. Three decision makers $d_{k}$ ( $k=1,2,3$ ) (whose weighting vector is

Table 4. Normalized decision matrix $\tilde{\boldsymbol{R}}^{(1)}$

\begin{tabular}{cccc}
\hline & $C_{1}$ & $C_{2}$ & $C_{3}$ \\
\hline$X_{1}$ & $\log -N\left(0.8797,0.0196^{2}\right)$ & $\log -N\left(0.8558,0.0242^{2}\right)$ & $\log -N\left(0.9353,0.0391^{2}\right)$ \\
$X_{2}$ & $\log -N\left(0.8931,0.0215^{2}\right)$ & $\log -N\left(0.8252,0.0261^{2}\right)$ & $\log -N\left(0.9559,0.0361^{2}\right)$ \\
$X_{3}$ & $\log -N\left(0.9538,0.0190^{2}\right)$ & $\log -N\left(0.8374,0.0209^{2}\right)$ & $\log -N\left(1.0000,0.0402^{2}\right)$ \\
$X_{4}$ & $\log -N\left(0.8201,0.0232^{2}\right)$ & $\log -N\left(0.9417,0.0230^{2}\right)$ & $\log -N\left(0.7831,0.0426^{2}\right)$ \\
$X_{5}$ & $\log -N\left(0.7581,0.0205^{2}\right)$ & $\log -N\left(0.9601,0.0212^{2}\right)$ & $\log -N\left(0.8176,0.0444^{2}\right)$ \\
\hline
\end{tabular}

Table 5. Normalized decision matrix $\tilde{\boldsymbol{R}}^{(2)}$

\begin{tabular}{cccc}
\hline & $C_{1}$ & $C_{2}$ & $C_{3}$ \\
\hline$X_{1}$ & $\log -N\left(0.9213,0.0205^{2}\right)$ & $\log -N\left(0.7699,0.0233^{2}\right)$ & $\log -N\left(0.9701,0.0325^{2}\right)$ \\
$X_{2}$ & $\log -N\left(0.9117,0.0217^{2}\right)$ & $\log -N\left(0.8252,0.0285^{2}\right)$ & $\log -N\left(0.9420,0.0361^{2}\right)$ \\
$X_{3}$ & $\log -N\left(0.9777,0.0198^{2}\right)$ & $\log -N\left(0.7761,0.0264^{2}\right)$ & $\log -N\left(0.9630,0.0385^{2}\right)$ \\
$X_{4}$ & $\log -N\left(0.7581,0.0232^{2}\right)$ & $\log -N\left(0.9479,0.0279^{2}\right)$ & $\log -N\left(0.7692,0.0444^{2}\right)$ \\
$X_{5}$ & $\log -N\left(0.7714,0.0207^{2}\right)$ & $\log -N\left(0.9785,0.0273^{2}\right)$ & $\log -N\left(0.8280,0.0509^{2}\right)$ \\
\hline
\end{tabular}

Table 6. Normalized decision matrix $\tilde{\boldsymbol{R}}^{(3)}$

\begin{tabular}{cccc}
\hline & $C_{1}$ & $C_{2}$ & $C_{3}$ \\
\hline$X_{1}$ & $\log -N\left(0.9261,0.0196^{2}\right)$ & $\log -N\left(0.7822,0.0242^{2}\right)$ & $\log -N\left(0.9924,0.0337^{2}\right)$ \\
$X_{2}$ & $\log -N\left(0.8977,0.0209^{2}\right)$ & $\log -N\left(0.8252,0.0282^{2}\right)$ & $\log -N\left(0.9559,0.0361^{2}\right)$ \\
$X_{3}$ & $\log -N\left(1.000,0.0226^{2}\right)$ & $\log -N\left(0.7883,0.0264^{2}\right)$ & $\log -N\left(0.9774,0.0396^{2}\right)$ \\
$X_{4}$ & $\log -N\left(0.9696,0.0226^{2}\right)$ & $\log -N\left(1.0000,0.0285^{2}\right)$ & $\log -N\left(0.7738,0.0420^{2}\right)$ \\
$X_{5}$ & $\log -N\left(0.7484,0.0249^{2}\right)$ & $\log -N\left(0.9479,0.0270^{2}\right)$ & $\log -N\left(0.8228,0.0450^{2}\right)$ \\
\hline
\end{tabular}


$\left.\boldsymbol{e}=(0.30,0.40,0.30)^{T}\right)$ evaluate the performance of these companies $X_{i}(i=1,2, \cdots, 5)$ with respect to the criteria $C_{j}(j=1,2,3)$ by $\log$-normally distributed random variables $\beta_{i j}^{(k)}=\log -N\left(\mu_{i j}^{(k)},\left(\sigma_{i j}^{(k)}\right)^{2}\right)$, and construct the decision matrices $\boldsymbol{R}^{(k)}=\left(\beta_{i j}^{(k)}\right)_{5 \times 3}(k=1,2,3)$ as listed in Table $1 \sim$ Table 3 .

To get the best company, the following steps are involved.

Step 1 Utilize Eq. (17) Eq. (19) to normalize the decision matrices $\boldsymbol{R}^{(k)}=\left(\beta_{i j}^{(k)}\right)_{5 \times 3}$ into the corresponding decision matrices $\tilde{\boldsymbol{R}}^{(k)}=\left(\tilde{\beta}_{i j}^{(k)}\right)_{5 \times 3}$ $(k=1,2,3)$ as listed in Table $4 \sim$ Table 6 . Note that the criterion $C_{2}$ is benefit criterion and the criteria $C_{1}$ and $C_{3}$ are cost criteria.

Step 2 Utilize Eq. (20) to aggregate the criterion values of the $i$ th column of the normalized decision matrices $\tilde{\boldsymbol{R}}^{(k)}=\left(\tilde{\beta}_{i j}^{(k)}\right)_{5 \times 3}$ and derive the individual overall values $\tilde{\beta}_{i}^{(k)}$ of the alternatives $X_{i}$ ( $i=1,2, \cdots, 5$ ) given by the decision makers $d_{k}$ $(k=1,2,3)$ :

$$
\begin{aligned}
& \tilde{\beta}_{1}^{(1)}=\log -N\left(0.8864,0.0157^{2}\right), \\
& \tilde{\beta}_{2}^{(1)}=\log -N\left(0.8855,0.0159^{2}\right), \\
& \tilde{\beta}_{3}^{(1)}=\log -N\left(0.9237,0.0152^{2}\right), \\
& \tilde{\beta}_{4}^{(1)}=\log -N\left(0.8547,0.0168^{2}\right), \\
& \tilde{\beta}_{5}^{(1)}=\log -N\left(0.8495,0.0163^{2}\right), \\
& \tilde{\beta}_{1}^{(2)}=\log -N\left(0.8790,0.0144^{2}\right), \\
& \tilde{\beta}_{2}^{(2)}=\log -N\left(0.8882,0.0165^{2}\right), \\
& \tilde{\beta}_{3}^{(2)}=\log -N\left(0.8990,0.0161^{2}\right), \\
& \tilde{\beta}_{4}^{(2)}=\log -N\left(0.8314,0.0181^{2}\right), \\
& \tilde{\beta}_{5}^{(2)}=\log -N\left(0.8639,0.0189^{2}\right), \\
& \tilde{\beta}_{1}^{(3)}=\log -N\left(0.8914,0.0147^{2}\right), \\
& \tilde{\beta}_{2}^{(3)}=\log -N\left(0.8871,0.0163^{2}\right), \\
& \tilde{\beta}_{3}^{(3)}=\log -N\left(0.9154,0.0168^{2}\right), \\
& \tilde{\beta}_{4}^{(3)}=\log -N\left(0.9260,0.0177^{2}\right), \\
& \tilde{\beta}_{5}^{(3)}=\log -N\left(0.8430,0.0183^{2}\right) .
\end{aligned}
$$

Step 3 Utilize Eq. (22) to derive the collective overall values $\tilde{\beta}_{i}$ of the alternatives $X_{i}(i=1,2, \cdots, 5)$, where the weighting vector of LNDHG operator is $\omega=(0.2429,0.5142,0.2429)^{T} \quad$ which is determined by the normal distribution based method ${ }^{35}$ :

$$
\begin{aligned}
& \tilde{\beta}_{1}=\log -N\left(0.8625,0.0087^{2}\right), \\
& \tilde{\beta}_{2}=\log -N\left(0.8630,0.0096^{2}\right), \\
& \tilde{\beta}_{3}=\log -N\left(0.8896,0.0092^{2}\right), \\
& \tilde{\beta}_{4}=\log -N\left(0.8577,0.0104^{2}\right), \\
& \tilde{\beta}_{5}=\log -N\left(0.8292,0.0102^{2}\right) .
\end{aligned}
$$

Step 4 Utilize Eq. (1) to calculate the expected values $\mu_{\log }\left(\tilde{\beta}_{i}\right)$ of the collective overall values $\tilde{\beta}_{i}$ $(i=1,2, \cdots, 5)$ :

$$
\begin{gathered}
\mu_{\log }\left(\tilde{\beta}_{1}\right)=2.3692, \mu_{\log }\left(\tilde{\beta}_{2}\right)=2.3704, \\
\mu_{\log }\left(\tilde{\beta}_{3}\right)=2.4343, \mu_{\log }\left(\tilde{\beta}_{4}\right)=2.3580, \\
\mu_{\log }\left(\tilde{\beta}_{5}\right)=2.2917 .
\end{gathered}
$$

Thus

$$
\begin{gathered}
\mu_{\log }\left(\tilde{\beta}_{3}\right)>\mu_{\log }\left(\tilde{\beta}_{2}\right)>\mu_{\log }\left(\tilde{\beta}_{1}\right)> \\
\mu_{\log }\left(\tilde{\beta}_{4}\right)>\mu_{\log }\left(\tilde{\beta}_{5}\right) .
\end{gathered}
$$

Step 5 Use Definition 2 to rank all the alternatives $X_{i}(i=1,2, \cdots, 5)$ :

$$
X_{3} \succ X_{2} \succ X_{1} \succ X_{4} \succ X_{5} .
$$

Therefore, the best investment enterprise is $X_{3}$.

\section{Conclusions}

In this paper, we have extended the WGA operator and the OWG operator to accommodate the stochastic uncertain situations where the given arguments take the form of log-normally distributed random variables, proposed some new geometric aggregation operators, such as the LNDWG operator, the LNDOWG operator and the LNDHG operator, and established various desirable properties of these operators. Weights represent different aspects in both the LNDWG operator and the LNDOWG operator. The LNDWG operator weights only the log-normal distributions, the LNDOWG operator weights only the ordered positions of the log-normal distributions instead of weighting the log-normal distributions themselves, and both these two operators consider only one of them. The LNDHG operator weights both the given log-normal distributions 
and their ordered positions, and generalizes both the LNDWG operator and the LNDOWG operator at the same time. In addition, the LNDOWG operator and the LNDHG operator can relieve the influence of unfair data on the final results by assigning low weights to those unduly high or unduly low ones. Furthermore, we have given an application of the LNDWG operator and the LNDHG operator to MCGDM based on lognormally distributed random variables. This paper enriches and develops aggregation operator theory. In the future, we shall continue working in the extension and application of the developed operators to other domains.

\section{Acknowledgements}

The authors are very grateful to the editor and the anonymous referees for their constructive comments and suggestions that have led to an improved version of this paper. This work was supported by the National Natural Science Foundation of China (No. 71271218, 71221061 and 61174075), and the Humanities and Social Science Foundation of the Ministry of Education of China (No. 12YJA630114 and 13YJC630200).

\section{References}

1. J. Aczél and T.L. Saaty, Procedures for synthesizing ratio judgments, Journal of Mathematical Psychology 27 (1) (1983) 93-102.

2. Z.S. Xu and Q.L. Da, The ordered weighted geometric averaging operators, International Journal of Intelligent Systems 17 (7) (2002) 709-716.

3. Z.S. Xu, A fuzzy ordered weighted geometric operator and its application in fuzzy AHP, Systems Engineering and Electronics 24 (7) (2002) 31-33.

4. Y.J. $\mathrm{Xu}$ and Q.L. Da, Uncertain ordered weighted geometric averaging operator and its application to decision making, Systems Engineering and Electronics 27 (6) (2005) 1038-1040.

5. X.F. Wang and X.J. Yang, Trapezoidal fuzzy ordered weighted geometric operator and its application to decision making, Mathematics in Practice and Theory $\mathbf{4 1}$ (20) (2011) 78-83.

6. Z.S. Xu and R.R. Yager, Some geometric aggregation operators based on intuitionistic fuzzy sets, International Journal of General Systems 35 (4) (2006) 417-433.

7. G.W. Wei, Some geometric aggregation functions and their application to dynamic multiple attribute decision making in the intuitionistic fuzzy setting, International Journal of Uncertainty, Fuzziness and Knowledge-Based Systems 17 (2) (2009) 179-196.

8. C. Tan, Generalized intuitionistic fuzzy geometric aggregation operator and its application to multi-criteria group decision making, Soft Computing 15 (5) (2011) 867-876.

9. Z.S. $\mathrm{Xu}$, Methods for aggregating interval-valued intuitionistic fuzzy information and application to decision making, Control and Decision 22 (2) (2007) 215-219.

10. Z.S. $\mathrm{Xu}$ and J. Chen, On geometric aggregation over interval-valued intuitionistic fuzzy information, in: Proceedings of Fourth International Conference on Fuzzy Systems and Knowledge Discovery (FSKD 2007), Haikou, China, 2007, pp. 466-471.

11. X.F. Wang, Fuzzy number intuitionistic fuzzy geometric aggregation operators and their application to decision making, Control and Decision 23 (6) (2008) 607-612.

12. Z.S. $\mathrm{Xu}, \mathrm{A}$ method based on linguistic aggregation operators for group decision making with linguistic preference relations, Information Sciences 166 (1-4) (2004) 19-30.

13. G.W. Wei, A method for multiple attribute group decision making based on the ET-WG and ET-OWG operators with 2-tuple linguistic information, Expert Systems with Applications 37 (12) (2010) 7895-7900.

14. Z.S. Xu, An approach based on the uncertain LOWG and induced uncertain LOWG operators to group decision making with uncertain multiplicative linguistic preference relations, Decision Support Systems 41 (2) (2006) 488-499.

15. G.W. Wei, Uncertain linguistic hybrid geometric mean operator and its Application to group decision making under uncertain linguistic environment, International Journal of Uncertainty, Fuzziness and Knowledge-Based Systems 17 (2) (2009) 251-267.

16. R. Lahdelma, S. Makkonen and P. Salminen, Multivariate Gaussian criteria in SMAA, European Journal of Operational Research 170 (3) (2006) 957-970.

17. S.B. Yao and C.Y. Yue, Approach to stochastic multiattribute decision problems using rough sets theory, Journal of Systems Engineering and Electronics 17 (1) (2006) 103-108.

18. G.T. Jiang, Z.P. Fan and Y. Liu, Method for multiple attribute decision making with normal random variables, Control and Decision 24 (8) (2009) 1187-1192.

19. Y. Liu, Z.P. Fan and Y. Zhang, A method for normal stochastic multiple attribute decision making considering interactions among attributes, Operations Research and Mangement Science 20 (5) (2011) 20-26.

20. G.T. Jiang and Z.P. Fan, A method for MADM with normal random variables, Systems Engineering-Theory \& Practice 32 (7) (2012) 1517-1524.

21. J.Q. Wang and J. Ren, Stochastic multi-criteria decisionmaking method based on WC-OWA operator, Control and Decision 22 (12) (2007) 1429-1432.

22. J. Ren and Y. Gao, Stochastic multi-criterion decisionmaking method based on interval operation, Systems Engineering and Electronics 32 (2) (2010) 308-312.

23. X.F. Wang and X.J. Yang, Dynamic stochastic multiple attribute decision making method with incomplete certain 
information, Systems Engineering: Theory \& Practice 30 (2) (2010) 332-338.

24. J.C. Harsanyi, Cardinal welfare, individualistic ethics, and interpersonal comparisions of utility, Journal of Political Economy 63 (4) (1955) 309-321.

25. A. Sen, Collective Choice and Social Welfare, HoldenDay, San Francisco, CA, 1970.

26. M. Nowak, Aspiration level approach in stochastic MCDM problems, European Journal of Operational Research 177 (3) (2007) 1626-1640.

27. K. Zaras, Rough approximation of a preference relation by a multi-attribute dominance for deterministic, stochastic and fuzzy decision problems, European Journal of Operational Research 159 (1) (2004) 196-206.

28. J. Aitchison and J.A.C. Brown, The Lognormal Distribution, Cambridge University Press, Cambridge, 1957.

29. E.L. Crow and K. Shimizu, Lognormal Distributions: Theory and Application, Dekker, New York, 1988.

30. E. Limpert, W. Stahel and M. Abbt, Log-normal distributions across the sciences: keys and clues, BioScience 51 (5) (2001) 341-352.

31. I. Antoniou, V.V. Ivanov, V.V. Ivanov, P.V. Zrelov, On the log-normal distribution of stock market data, Physica A: Statistical Mechanics and its Applications 331 (3-4) (2004) 617-638.

32. R.C. Merton and P.A. Samuelson, Fallacy of the lognormal approximation to optimal portfolio decisionmaking over many periods, Journal of Financial Economics 1 (1) (1974) 67-94.

33. L.W. Chen, Project Investment Risk Analysis: Theories and Methods, China Machine Press, Beijing, 2004.

34. G. McPherson, Statistics in scientific investigation: its basis, application and interpretation, Springer-Verlag, New York, 1990.

35. Z.S. Xu, An overview of methods for determining OWA weights, International Journal of Intelligent Systems 20 (8) (2005) 843-865. 